\title{
IX.
}

\section{Einige wenig beschriebene Formen der Tabes dorsalis.")}

\author{
Von \\ Michael Lapinsky, \\ Professor an der St. Wladimir-Universität zu Kiew.
}

Unter den zahlreichen Erkrankungen des Rückenmarks galt die Tabes dorsalis immer als eine Erkrankung vorzüglich des sensiblen Apparates. Die Anfangssymptome dieser Erkrankung bestehen, wie es aus den Beschreibungen aller Autoren hervorgeht, in verschiedenartigen Paräthesien, Schmerzempfindungen, Verlust der Schmerz-, Temperatur- und Tastempfindung und des Muskelsinnes; späterhin werden, infolge derselben Affektion des sensorischen Neurons, die einfachsten Reflexe und die komplizierten reflektorischen Akte gestört; die motorische Sphäre aber bleibt lange Zeit über normal. Ein so gewiegter Kenner der Erkrankungen des Nervensystems wie Oppenheim äussert bei der Besprechung der Störungen dieser Sphäre seine Meinung in dem Sinne, dass die Veränderungen der Bewegungen bei der Tabes dorsalis - soweit es die Extremitäten betrifft - in einer Störung der Koordination, nicht aber in Schwäche bestehen. Die einzelnen Bewegungen des Fusses oder der Hand kann der Patient in vollem Umfange und mit genügender Kraft ausführen, aber der gewünschte Willensakt wird nicht sofort und auf dem kürzesten Wege ausgeführt, sondern das gesteckte Ziel wird erst nach wiederholten Korrekturen und unter starker Inanspruchnahme der Energie erreicht (Lehrbuch der Nervenkrankheiten, Berlin 1898. S. 125).

Indem ich mich mit dem soeben zitierten Autor insofern einverstanden erkläre, als sich seine Bemerkungen auf die Mehrzahl der Tabetiker beziehen, will ich mir erlauben, hier einige Fälle von Tabes dorsalis anzuführen, in denen die primären Krankheitssymptome mehr in der motorischen als in der sensiblen Sphäre zutage traten.

1. M. A. K .....zewa, 23jähriges Mädchen, gehört einer neurapathischen Familie an. Die Mutter der Patientin leidet an Neurasthenie

*) Vorgetragen in der Sitzung der Alexander-Hospital-Ärzte. 
und Arthritis. Ihr Vater war in seinen ersten Jünglingsjahren an Syphilis erkrankt. Zwei Schwestern der Patientin leiden in leichter Form an Hystero-Neurasthenie. Die Patientin selbst litt in den letzten Jahren ihrer Schulzeit an Schwindel, Herzklopfen und Schlaflosigkeit. Mehrfach traten bei ihr nach starken Aufregungen hysterische Anfälle auf. In den letzten Jahren wiederholten sich derartige Anfälle nicht mehr und die Patientin widmete ihre freie Zeit der Bibliothek der Gesellschaft für Volksbildung und einigen Sonntagsschulen. Beim Gespräch mit Frl. K. Kommt man zu der Überzeugung, dass sie eine überlegende, vernünftige Persönlichkeit ist, der Prahlerei und Koketterie fremd sind and die wenig eindrucksfähig ist.

Im April 1901, als ich zum ersten Mal zur Patientin gebeten wurde, fand ich dieselbe im Bett liegend vor. Die Klagen der Patientin bestanden in Folgendem: Ungefähr 3 Wochen vor meinem Besuch fühlte Patientin, beim Aufheben eines schweren Bücherpackets, zum ersten Mal eine Schwäche im linken Fuss; eine Woche darauf stellte sich eine ebensolche Schwäche iu der rechten Hand ein. Von diesem Zeitpunkte an progressierten diese Schwächeerscheinungen immer mehr und mehr und steigerten sich bis zum Grade einer Parese; in den letzten zwei Tagen konnte Patientin gar nicht mehr gehen and konnte nur mit Mühe die rechte Hand beim Ankleiden, Essen und anderen groben Bewegungen benutzen; Bewegungen hingegen, wie z. B. Nähen und Schreiben, waren nicht gestört. Schmerzen und Paräthesien hat Patientin nicht bemerkt. Der Schlaf ist gut, die Gemütsstimmung ist eine rubige. Erkältungen, Kontusionen und andere Schädlichkeiten sind in den letzten 4 Wochen nicht vorgekommen. 5 Monate ror den beschriebenen Begebenheiten starb plötzlich der Vater der Patientin; der Tod des Vaters verursachte der Patientin einige Zeit hindurch seelisches Leiden, bald jedoch widmete sie sich wieder ihren gewohnten Beschäftigungen und beruhigte sich allmählich vollständig.

Die sorgfältige objektive Untersuchung ergab Folgendes:

Kein Ödem der Extremitäten; keine Anschwellangen der Gelenke; die Gelenke knirschen nicht und sind nicht schmerzhaft.

Der linke Fuss befindet sich im Zustande einer tiefen Parese, wobei die Zehen dem Willen absolut nicht gehorchen; im Fussgelenk sind die willkürlichen Bewegungen nur sehr gering und werden mit grossem Aufwand von Energie und nur sehr langsam ausgeführt. Die rechte untere Extremität weicht im Sinne der motorischen Funktionen nicht von der Norm ab; die linke obere Extremität ist in dieser Beziehung ebenfalls normal; die rechte obere Extremität ist paretisch und die rohe Kraft ist her'abgesetzt.

Am linken Auge besteht deutlich ausgesprochene Ptosis.

Die taktile Empfindung ist am Rumpf und an den Extremitäten überall normal. Die Schmerzempfindung ist in verschiedener Weise verändert. Auf dem rechten Fussrücken waren Erscheinungen von Hyperästhesie vorhanden, am linken Fussrücken war die Schmerzempfindung etwas herabgesetzt und zwar an der rechten unteren Extremität weniger als an der linken; auf der vorderen äusseren Oberfläche des Unterschenkels war sie ebenfalls stärker herabgesetzt als auf der hinteren Seite. Die linke Hälfte des Abdomens ist in Bezug auf die Schmerzempfindung normal, auf der rechten Hälfte ist dieselbe stark herabgesetzt. Das Gebiet des 3., 4. und 5. Interkostalraumes war anf beiden Seiten in gleichem Grade 
hyperästhetisch. Die rechte obere Extremität war im Gebiet der 5. und 6. hinteren Wurzeln hyperästhetisch. Die linke obere Extremität, der Hals und das Gesicht wichen von der Norm nicht ab. Der Muskelsinn war durchaus normal. (Die Temperaturempfindung wurde bei dieser ersten Untersuchung nicht bestimmt. Der Gang und das Steigen wurde ebenfalls nicht untersucht, da die Patientin zu Bett lag). Die Haut- und Sehnenreflexe waren alle vorhanden und zwar erhöht. Der Pharyngealreflex war normal. Die Lichtreaktion der Pupillen war ebenfalls normal. Die peripheren Nervenstämme waren nicht im geringsten druckempfindlich. Der Umfang der Muskeln war durchaus unverändert, ihr Tonus war aber bemerkbar herabgeseszt. Beide Nieren liessen sich nicht palpieren und waren nicht schmerzhaft. Starke Abmagerung, Herabsetzung des Ernährungszustandes und Blutarmut konnten nicht konstatiert werden. Die faradische und galvanische Reizbarkeit der Nerven and Muskeln war normal. Die Diagnose war in diesem Falle nicht mit Sicherheit zu stellen. Mit Sicherheit konnten Rheumatismus, Myositis and Neuritis ausgeschlossen werden; die Schmerzen fehlten, die Gelenke waren ebenso wie die Muskulatur und die peripheren Nerven bei Druck nicht empfindlich; die elektrische Erregbarkeit war ebenfalls normal. Ebenso kounten in diesem Falle Poliomyelitis anterior acuta oder subacuta, die verschiedenen Formen von Muskelatrophie und Sclerosis lateralis amyotrophica ausgeschlossen werden, denn gegen diese Erkrankungsformen sprachen das Fehlen von Atrophien und das Vorhandensein von Analgesien, Hypalgesien und Hyperästhesien.

In Anbetracht dessen, dass die Patientin einer neuropathischen Familie angehört, dass zwei von ihren Schwestern an Hystero-Neurasthenie leiden und dass in der Anamnese "hysterische Anfälle" verzeichnet sind, wurde, freilich ohne vollkommen einwandsfreie Begründung, die Diagnose Hysterie gestellt und die Patientin wurde der hydropathischen Anstalt von Dr. A. $\mathrm{N}$. Uspensky überwiesen, wo ihr Duschen, faradischer Strom zur Behandlung der paretischen Muskeln und statische Elektrizität verordnet wurden.

Als ich die Patientin nach 3 Monaten zum zweiten Mal beswchte, war sie nicht mehr bettlägerig; sie konnte, wenn auch nur mit grosser Anstrengung, gehen und klagte über Schwäche im rechten Fuss und in der linken Hand; gehen kann Patientin nur am Tage, in der Dunkelheit geht sie sehr unsicher. In den letzten 3 Wochen hat die Patientin bemerkt, dass sie beim Harnlassen drängen muss; Schmerzen empfindet sie nirgends im Körper.

Bei der objektiven Untersuchung fand ich jetzt Folgendes: Die rohe Kraft der rechten oberen Extremität steht stark hinter der der linken zurück. An der linken unteren Extremität ist die rohe Kraft noch bedeutender herabgesetzt, die Patientin kann aber ihre Zehen und den Fuss frei bewegen. Sie kann gehen und sogar Treppen steigen. Die Gangart ist stark verändert, die Beine sind gespreizt und werden von der Patientin beim Gehen zur Seite geworfen; beim Gehen schwankt die Patientin und hält mit den Händen das Gleichgewicht, indem sie mit denselben in der Luft balanziert. Mit geschlossenen Augen kann Patientin weder gehen noch stehen. Auf einem Fuss kann sie nicht stehen. Die Ptosis ist verschwunden. Die Veränderungen der Empfindung sind nur am Abdomen verschwunden. An den unteren Extremitäten, in den Interkosstalräumen 
und an der linken Hand befinden sie sich im Status quo ante. Die Hautund Sehnenreflexe sind wie bisher erhöht. Der Pupillenreflex ist etwas matt. Im Anfang Oktober 1901, d. h. 7 Monate nach meinem ersten Besuch, untersuchte ich die Patientin noch einmal. Die Klagen der Pat. beștanden wie bisher in Schwächegefühl, obgleich in bedeutend geringerem Grade als vorher; während sie vorher nur eine Schwäche in einem Fuss erwähnte, bemerkte sie jetzt auch eine Schwäche im anderen Fuss. In der Dunkelheit und mit geschlossenen Augen kann Patientin nicht gehen. In den Füssen hat sich ein unbestimmtes Vertaubungsgefühl eingestellt und Patientin hat das Gefühl, als ob sie auf einem weichen, dicken Teppich ginge. (Die Symptome von Ataxie haben sich, nach Aussage der Patientin, in den letzten 2 Monaten entwickelt). Am Abdomen und dem Brustkorb hat sie das Gefühl, als ob diese Körperteile von einem Korsett oder Gurt umschlossen würden. Irgend "welche - speziell ausstrahlende Schmerzen verspürt Patientin nicht. Das Drängen beim Harnlassen ist nicht mehr so ansgesprochen, jedenfalls hat Patientin zuweilen keinerlei Beschwerden in dieser Beziehung. Zuweilen sieht Patientin die Gegenstände doppelt. Die Gemütsstimmung ist eine ruhige, der Schlaf gut.

Die objektive Untersuchung ergab Folgendes:

Die rohe Kraft der beiden unteren Extremitäten ist in gleichem MaBe herabgesetzt; der Muskeltonus ist matt; ihr Umfang normal. Atrophien und fibrilläres Zucken sind nirgends vorhanden. Die peripheren Nerven sind nirgends schmerzhaft; die Gelenke sind ebenfalls nicht schmerzhaft, die passiven Bewegangen gehen in denselben vollkommen frei von statten, und in den Gelenken ist keine Krepitation bemerkbar. Die faradische und galvanische Erregbarkeit der Nerven und Muskeln ist normal. Der Gang ist ataktisch. Patientin geht breitbeinig und unsicher. Die Fussspitzen werden stark nach aussen gesetzt. Rombergsches Symptom. Beim Konvergieren der Sehaxen tritt ein Abweichen des linken Auges nach aussen hin ein und die Patientin sieht den fixierten Gegenstand doppelt.

Die Tastempfindung zeigt keine besonderen Abweichungen von der Norm. Was die Schmerzempfindung anbetrifft, so bestehen eine seharf ausgeprägte Hyperästhesie der Fussrücken beider Füsse und volle Analgesie der Sohle und im Insertionsgebiete der Achillessehne; einzelne analgetische Stellen sind auch am Planum tibiae der linken Seite bis zum unteren $1 / 4$ des Oberschenkels und auf der rechten Seite an der hinteren Seite des Oberschenkels und an der Wade vorhanden.

Die Schmerzempindung des Abdomens ist normal. Auf beiden Seiten der Brust zeigen einzelne Interkostalräume bald Hyperästhesie, bald hingegen Hypalgesie.

An der linken oberen Extremität ist das Gebiet der Ausbreitung der 5. hinteren Halswurzel für Nadelstiche fast vollständig unempfindlich, auch im Gebiet der 8. hinteren Hals- und der 1. hinteren Brustwurzel ist die Schmerzempfindung herabgesetzt. Die Schmerzempfindung im Ausbreitungsgebiete der übrigen hinteren Wurzeln weicht nicht von der Norm $a b$.

Die rechte obere Extremität zeigt Erscheinungen von Hypalgesie im، Ausbreitungsgebiet der 7. und 8. hinteren Halswurzeln, die ubbrigen Teile der oberen Extremität sind normal. 
Der Muskelsinn ist an den unteren Extremitäten herabgesetzt. Die Patientin versieht sich bei der Angabe der Lage der Zehen; sie sucht lange das Knie mit dem Hacken, wenn man sie dazu auffordert den letzteren auf das Knie zu legen.

Die Haut- and Sehnenreflexe sind überall erhöht. Der Pharyngealreflex weicht nicht von der Norm ab. Die Pupillen sind ungleich und reagieren auf Lichtwechsel nur matt. Sowohl die faradische als auch die galvanische Reaktion ist vollständig unverändert.

Den Herbst und Winter verbrachte Patientin am Ufer des schwarzen Meeres, wo sie an Stelle der verordneten Jodkur Galvanisation der Wirbelsäule, Faradisation der Haut und halbwarme Duschen anwandte. Nach ihrer Rückkehr im März 1902 klagte Patientin über scharf ausgeprägte Ataxie, Korsett- und Gürtelgefühl und Drängenmüssen beim Harnlassen. Von Zeit zu Zeit zeigten sich ausstrahlende Schmerzen.

Im April 1903 ergab die objektive Untersuchung eine Herabsetzung der Tastempfindung an den unteren Extremitäten und dem Rumpf bis zur Höhe der Brustwarzen. Die Schmerzempfindnng ist, den Gebieten der hinteren Wurzeln entsprechend, gestört. Das Gebiet der 5. Lendenwurzel zeigte auf beiden Seiten eine scharf ausgeprägte Steigerung der Schmerzund Temperaturempfindung; eine ebensolche Steigerung der Schmerzempfindung wurde links im Gebiet der 1. Lendenwurzel und der 3.-5. Kreuzbeinwurzeln konstatiert. Alle übrigen Zonen der unteren Extremitäten waren entweder für Nadelstiche vollständig unempfindlich oder die Schmerzempfindung war stark herabgesetzt. Die Schmerzempfindung am Hacken zeigt ausser einer Herabsetzung eine Verlangsamung der Weiterleitung und gleichzeitig hiermit eine verstärkte Nachempfindung des Schmerzes. Eine Hyperästhesie für Nadelstiche war auch im Gebiet des 3. und 4. Interkostalnerven am Rumpf und im Gebiet der 5. und 8. hinteren Halswurzeln an der linken Hand zu bemerken. Die übrigen Teile besassen ein normales Empfindungsvermögen. Der Achillesreflex war am linken Fuss äusserst matt und fehlte am rechten Fuss vollständig. Die übrigen Haut- und Sehnenreflexe zeigten keine Abweichungen von der Norm. Die galvanische und faradische Reaktion waren normal. Die Nerven sind bei Druck nicht empfindlich. Atrophien und fibrilläres Zittern sind nicht vorhanden. Der Muskeltonus der unteren Extremitäten ist matt. Der Muskelsinn befindet sich im Status quo ante. Die Gangart ist ataktisch. Mit geschlossenen Augen kann Patientin überhaupt nicht gehen.

Die Patientin warde nach dreiwöchentlicher Behandlung mit der faradischen Bürste (mit zunehmender Spannung) der Wasserheilanstalt von Dr. Frenkel in Kiew übergeben, wo sie mit Wannen, mit der faradischen Bürste, mit Galvanisation und Kauterisation der Wirbelsäule behandelt wurde.

Diese Behandlungsmethode erzielte im Verlauf von 8 Wochen eine vollständige Wiederherstellung der Tastemptindung und eine bedeutende Besserung der Empfindung für Nadelstiche und des Muskelsinns. Die Ataxie hat sich bedeutend vermindert, so dass Patientin mit geschlossenen Augen breitbeinig stehen und 10-15 Schritte gehen kann.

Die Hyperästhesie und Analgesie besteht in den früher konstatierten Zonen, die von den hinteren. Wurzeln versorgt werden, in gleicher Weise fort. Die fehlenden oder matten Reflexe der Achillessehne blieben eben- 
falls ohne Besserung. Die rohe Kraft ist an den unteren Extremitäten noch immer herabgesetzt.

Die Sommersaisons von 1904 und 1905 verbrachte Patientin in Nauheim, da die Schmerzempindung wieder abgenommen and die Ataxie sich verstärkt hatte. Nach dem Aufenthalt in Nauheim wurde die Empfindung besser, die rohe Kraft der Füsse blieb aber nach wie vor eỉne geschwächte.

Die hier in kurzen Zügen angeführte Krankengeschichte weist zweifellos auf einen Fall von Tabes dorsalis hin. Hierfür spricht der typische ataktische Gang, das Rombergsche Symptom, die Verteilung derStörungen der Schmerzempfindung in Form von Hyperalgesien und $\mathrm{H}_{y-}$ poalgesien, die Verlangsamung der Weiterleitung der Empfindung und die verstärkte Nachempfindung des Schmerzes, ferner das Korsett- und Gürtelgefühl, die Parästhesien in den Füssen, das Drängenmüssen beim Harnlassen, die matten Pupillenreflexe und die ungleichmässige Breite derselben, der matte Achillesreflex auf der einen Seite und das Fehlen dieses Reflexes auf der anderen Seite. Alle diese Symptome lassen keinen $Z_{w}$ eifel daran aufkommen, dass wir das ataktische Stadium von Tabes dorsalis vor uns haben. In der präataktischen Periode aber, als das Krankheitsbild in einer gekreuzten Parese der oberen und unteren Extremität bestand, die so scharf ausgesprochen war, dass die Zehen vollständig paralysiert waren, dominierten die motorischen Störungen über alle übrigen Symptome. In dieser Periode konnte man durchaus nicht ein Leiden des Rückenmarks in Form von Tabes annehmen und dennoch bestand dieses Leiden schon damals, wie es aus der weiteren Beobachtung der Patientin ersichtlich ist.

2. M. W. D . . . kowsky, 37jähriger Techniker, wandte sich am 4. III. 1902 an mich mit folgenden Klagen:

Schon seit $1 \frac{1 / 2}{2}$.Jahren fühlt sich Patient nicht mehr ganz wohl. Die ersten Anzeichen seines Unwohlseins bestanden in Müdigkeit und Schwächegefühl in beiden Beinen, stärker war dieses Gefühl am linken Bein ausgesprochen. Dieses Gefühl stellte sich ohne besondere vorausgehende Ursachen ein. Nach 2 oder 3 Monaten fühlte D. ein unangenehmes Spannungsgefühl im Unterschenkel und zwar war das Gefühl derart, als ob der Unterschenkel mit einem engen Strumpf bekleidet wäre. Diese Empfindungen waren nicht sehr stark ausgeprägt und angenscheinlich beachtete D. dieselben auch nur wenig und hält die Schwäche in den Beinen für den Beginn seiner Erkrankung; jedenfalls lässt sich in der Anamnese das Vorhandensein von Paräthesien nur bei sorgfältigem Ausfragen and durch Erinnern von seiten des Arztes in Erfahrung bringen.

Nach 10 Monaten bemerkt Patient ein Abnehmen der geschlechtlichen Funktionen und eine Störung beim Harnlassen - der Harn fliesst sehr langsam ab und zur Beschleunigung beim Harnlassen wird die Beteiligung der Bauchpresse erforderlich. Ende 1901 bemerkt D. Schwierigkeiten beim Gehen im Dunkeln and gleichzeitig hiermit macht sich zeitweilig ein Doppeltsehen von fixierten Gegenständen bemerkbar. Alle diese aufeinander 
folgenden neuen Symptome treten im allgemeinen Krankheitsbilde in den Hintergrund and nach wie vor dominiert der Kräfteverfall der unteren Extremitäten. Diese Schwäche beunruhigt den Patienten hauptsächlich dadurch, dass er gleichzeitig eine Abmagerung der Füsse bemerkte, die anfangs progressierte, dann, ohne dass irgend welche therapeutischen Massnahmen angewandt worden wären, stehen blieb und dann von einer Zunahme der Füsse, ohne Zunahme der Kräfte in denselben, abgelöst wurde.

Von früheren Erkrankungen und Schädlichkeiten erwähnt D. häufige Erkältungen der Beine. In Bezug auf Syphilis kann er nichts Bestimmtes aussagen. Als sich das Doppeltsehen von Gegenständen bemerkbar machte, hat Patient aber auf Anraten des Arztes 80 Einreibungen von je $31 / 2 \mathrm{~g}$ grauer Quecksilbersalbe ausgeführt and 140 Gramm Natrium jodatum ausgetrunken. Seit dieser Behandlung sind ungefähr 4 Monate verflossen, irgend eine Besserung seines Zustandes und speziell des Doppeltsehens hat D. nicht bemerkt und, wie schon gesagt, progressierte die Schwäche in den Füssen immer mehr.

Bei der objektiven Untersuchung des Patienten am 4. III. 1902 wurde Folgendes konstatiert: Scharf ausgesprochene Abmagerung. Ödeme sind nicht vorhanden. Schwellung und Schmerzhaftigkeit der Gelenke ist nicht zu bemerken. Deformationen infolge von Muskelschwund sind nicht bemerkbar, die linke untere Fxtremität ist aber in ihrer ganzen Ausdehnung dünner als die rechte. Fibrilläres Zittern ist nirgends vorhanden. Der Muskeltonus der oberen Extremitäten und des Rumpfes ist normal. Der Muskeltonus der unteren Extremitäten ist stark herabgesetzt.

Die rohe Kraft der oberen Extremitäten weicht nicht stark von der Norm ab. Die rohe Kraft der unteren Extremitäten ist ungleichmässig herabgesetzt.

Bewegungen der linken unteren Extremität sind in allen Gelenken und in allen Richtungen möglich, diese Bewegungen sind aber weniger energisch und die kontrahierten Muskeln widerstehen dem Gegendruck nicht genügend. Als besonders schwach erweisen sich die kleinen Muskelgruppen beider Füsse.

Die rechte untere Extremität ist in toto stark paretisch. Während die Muskeln des Hüftgelenkes nur geschwächt, sind die Extensoren des Fusses und der Zehen fast vollständig paralysiert, so dass ein Heben des Fusses und der Zehen nicht möglich ist. Die Funktionen der übrigen Muskelgruppen des rechten Beines, darunter auch der M. quadriceps, sind tief paretisch. Beim Gehen stützt sich Patient auf das Bein, dasselbe knickt aber dabei im Knie ein. Beim Vorwärtsschreiten hebt D. den Fuss nicht nach oben, wie es bei einer Paralyse des $\mathrm{N}$. peroneus geschieht, sondern schleppt den Fuss nach, wie es bei Paralysen des MI. quadriceps und der Bengemuskeln des Oberschenkels geschieht.

Die Tastempfindung ist an den distalen Teilen beider Beine und in den Interkostalräumen etwas herabgesetzt. Die Schmerz- und Temperaturempfindung ist an der rechten unteren Extremität, ohne besonders genaue Grenzen, bedeutend herabgesetzt, bessert sich allmählich in der Richtung nach oben hin und wird in der Hüftgegend und in den unteren Teilen des Abdomens wieder normal. Das linke Bein ist in dieser Hinsicht normal, eine Ausnahme macht hierbei nur die Wadengegend und der Fussrücken, wo die Schmerz - und Temperaturempfindung mässig herabgesetzt ist. 
An beiden oberen Extremitäten besteht eine Herabsetzung der Temperatur- und Schmerzempfindung an der Innenseite im Gebiet der 1. und 2. Nervenwurzel der Brust. Ausserdem sind die rechte Schulter und das Gebiet des rechten Schulterblattes für Temperaturreize wenig empfindlich.

Der Muskelsinn ist an den unteren Extremitäten stark gestört; an den oberen Extremitäten weicht er nicht von der Norm ab.

Die Hautreflexe sind normal.

Die Sehnenreflexe und zwar der Achillessehnenreflex, der Patellarreflex, der Reflex der Tendo $m$. supinatoris longi und des M. biceps fehlen auf beiden Seiten vollständig.

Die rechte Pupille ist weiter als die linke; bei Lichtreiz bleiben beide vollständig unbeweglich; die sympathische Reaktion fehlt ebenfalls.

Die faradische und galvanische Reaktion weichen an beiden unteren und oberen Extremitäten von der Norm ab.

Die Daten der Anamnese lassen ein hereditäres Leiden des Muskelsystems ausschliessen. In der Anamnese sind aber Hinweise auf Syphilis der Eltern vorhanden (häufige Aborte bei der Mutter des Patienten).

Die hier beschriebene Erkrankung kann nicht für eine periphere Neuritis angesehen werden, da die elektrische Reaktion des faradischen und galvanischen Stromes normal ist und die Nervenstämme und Muskelmassen bei Druck wenig empfindlich sind. In gleicher Weise kann bei diesem Leiden nicht ron einer Poliomyelitis anterior oder einer spinalen Form von Muskelatrophie die Rede sein, da dem Vorhandensein dieser Erkrankungsformen die verschiedenen Empfindungsstörungen widersprechen.

Die Erkrankung konnte auch nicht durch ein Gelenkleiden erklärt werden, da die letzteren normal waren.

Die Abmagerung der erkrankten Extremität erlaubte auch nicht die Annahme einer hysterischen Paralyse.

Neben diesen zweifelhaften Annahmen waren aber unbestreitbare Anzeichen von Tabes dorsalis vorhanden und diese Diagnose musste auch für passend erachtet werden, wenn man die Ataxie, die Symptome von Robertson, Romberg und Westphal, die Empfindungsstörungen usw. berücksichtigt:

Patient wurde der Wasserheilanstalt von Dr. Us pensky überwiesen, wo eine Behandlung der unteren Extremitäten mit der faradischen Bürste, Halbbädern und Kauterisation der Wirbelsäule angewandt wurde. Ausserdem wurden Patienten Pillen aus Argentum nitricum und Secale cornutum verordnet.

Am 6. Juni 1903 erschien Patient wieder in meiner Sprechstunde.

Diesmal ergab die objektive Untersuchung Folgendes: Die Tast-, Temperatur- und Schmerzempfindung waren im allgemeinen und ganz besonders an den unteren Extremitäten bedeutend besser geworden, and Patient konnte, ohne Fehler dabei zu machen, kalte Gegenstände 
von warmen unterscheiden, was für ihn im März vorigen Jahres unmöglich war.

Der Muskelsinn, die Sehnenreflexe und die Reflexe der Pupillen waren vollständig unverändert geblieben. Die motorische Sphäre der rechten Extremität hatte sich hingegen sehr wesentlich vermindert. Das rechte Bein, dessen rohe Kraft im März mässig herabgesetzt war, war jetzt vollständig paretisch Am linken Bein, das sich früher in tiefer Parese befunden hatte, funktionierten jetzt alle, selbst die distalen Muskelgruppen, wenn auch noch etwas schwach. Der Muskeltonus war zweifellos höher als vor der Behandlung.

Der gegenwärtige Fall ist also ein typischer Fall von Tabes dorsalis in der präataktischen Periode dieser Erkrankung.

Die dominierenden Symptome dieses Leidens bestanden in den präataktischen Anfangsstadien, wie sich aus der Anamnese und dem Status praesens schliessen lässt, in paretischen und paralytischen $\mathrm{Er}$ scheinungen an den unteren Extremitäten.

3. Pet. ow, 28jähriger Offizier, erkrankte vor 9 Jahren an Syphilis. Im Verlauf der ersten 2 Jahre nach der Infektion führte er 5 Kurse einer spezifischen Kur durch (3 Kurse von Einreibung grauer Salbe und 2 Injektionskurse). 6 Jahre nach der Erkrankung fing Patient an zu schielen; nach 12 maligem Einreiben von grauer Salbe und nach 30 Injektionen hörte aber das Doppeltsehen auf. Im achten Jahre nach der syphilitischen Infektion, und zwar im Jahre 1903, begann Patient eine Schwäche in den Händen und Füssen zu fühlen. Früher war Patient ein guter Gymnastiker, war sehr gewandt und besass bedeutende Kraft in den Händen, jetzt ist er nicht mehr imstande, eine Volte auf dem Trapez auszuführen, sich mit den Händen an einer Leiter heraufzuziehen, Veloziped zu fahren und eine schräge Ebene hinaufzuklettern. Gleichzeitig fühlte er sich vollständig frisch and seine Gemütsstimmung liess nichts zu wünschen übrig. Patient fühlte keinerlei Schmerzen in den Gelenken der Extremitäten noch sonst irgendwo im Körper, die Funktionen der Geschlechtsorgane, des Darmes und der Harnblase wichen nicht von der Norm ab. Der Ernährangszustand hatte sich nicht verändert; eine Abmagerung hat Patient nicht bemerkt. Der Patient nahm an, dass die von ihm bemerkte Schwäche mit der früher durchgemachten syphilitischen Erkrankung im Zusammenhang stünde. Pat. wandte sich an den Arzt, der inn früher behandelt hatte und führte, auf Anraten des letzteren, zwei energische Kuren von je 24 Einreibungen von 6,0 grauer Salbe aus und brauchte innerlich gegen 100,0 Natrium jodatum.

Diese Therapie, auf die so viel Hoffnung gesetzt worden war, gab aber durchaus kein Resultat. Während der Behandlung nahm die Schwäche nicht ab, sondern im Gegenteil zu. Nach einiger Zeit bemerkte Patient noch ausserdem Beschwerden beim Harnlassen. Im Verlauf einiger Tage spürte Patient ausstrahlende Schmerzen in den Beinen, die aber von selbst wieder aufhörten.

Am 10. Februar 1904 wurde Pat. in die Nervenklinik am Alexanderkrankenhause aufgenommen. 
Der Krankengeschichte entnehme ich folgende kurze Daten:

Pat. ist von mittlerem Wuchs, normal gebaut und von gutem Ernährungszustand. Die Hautdecken zeigen keinerlei Abweichungen von der Norm. Ödem der Extremitäten ist nicht vorhanden. Die Lymphdrüsen lassen sich nicht palpieren; die Gelenke sind nicht schmerzhaft und sind frei beweglich. Die rohe Kraft der Extremitäten ist stark herabgesetzt und zwar ganz besonders an den unteren Extremitäten. Pat. kann nur 5 Schritte, auf einen Stock gestützt, machen und lässt sich dann kraftlos auf einen Stuhl sinken. Beim Gehen schleift Pat. beide Füsse auf der Diele. Ein besonders anffallendes Seitwärtsschleudern der Beine ist nicht zu bemerken. Im Bett liegend kann Pat. das Knie nicht bengen, wenn der untersuchende Arzt mit dem Zeigefinger auf den Oberschenkel oder den Unterschenkel drückt. Die im Gelenk gebeugte obere oder auch untere Extremität kann ohne jede Anstrengung passiv wieder gestreckt werden. Eine ebensolche Schwäche ist in den Bauchmuskeln bemerkbar. Im Gegensatz hierzu ist die Muskulatur des Rückens, des Halses und des Gesichtes vollständig normal. Das Rombergsche Symptom ist deutlich ausgesprochen. Die Tastempfindung ist vollständig normal.

Die Schmerz- und Temperaturempfindung sowie der Muskelsinn sind nor an den unteren Extremitäten und zwar an den distalen Teilen derselben mässig herabgesetzt.

Die Hautreflexe sind am Abdomen normal. Der Kremasterreflex und die Fersenreflexe fehlen. An der oberen Extremität sind der Ulnarreflex und der Sehnenreflex des M. triceps sehr matt; der Achillessehnenreflex und der Patellarreflex fehlen vollständig.

Der Pharyngealreflex ist normal.

Beim Harnlassen muss Patient drängen und entleert den Harn in einzelnen Portionen.

Die linke Pupille ist weiter als die rechte. Beide Pupillen reagieren auf Lichtreiz nicht; bei der Akkomodation und Konvergenz ist eine Verengerung der Pupillen bemerkbar.

Die faradische und galvanische Reizbarkeit der Nerven und Maskeln weicht nicht von der Norm ab. Bei Druck sind weder die Nerven, noch die Muskeln schmerzhaft.

Die vor der Aufnahme in die Nervenklinik ausgeführte Harnanalyse ergab nur Spuren von Quecksilber, infolge dessen wurden während des Anfenthalts des Patienten in der Klinik (er verweilte in derselben 4 Monate lang) noch 26 Einreibungen vorgenommen; die ganze Zeit über nahm Pat. Kali jodatum bis zu 3,0 pro die ein.

Gleichzeitig wurden die Muskeln des Patienten mit einer bipolaren Rolle faradisiert; auf die Wirbelsäule wurde ein aufsteigender galvanischer Strom von 5- 10 M.-A. appliziert. Ausserdem wurden noch Halbbäder und gymnastische Übungen nach Frenkel angewandt.

Alle diese verschiedenen therapeutischen Massnahmen brachten nur einen geringen Nutzen. An den Unterschenkeln besserte sich die Schmerzempfindang ein wenig, die Temperaturempfindung hingegen blieb unverändert. Die grobe Kraft der unteren Extremitäten nahm etwas zu, so dass der Patient auf ebener Diele, ohne sich zu erholen, allerdings auf einen Stock gestützt, ungefähr 40 Schritte machen konnte. Der Gang behielt aber seinen ataktischen Charakter bei. Was die Funktion der Harnblase 
anbetrifft, so hatte sich das Verlangen beim Harnlassen zu drängen bedeutend gebessert, Patient konnte aber am Morgen dennoch nur in hockender Stellung Harn lassen.

Mit Beginn der Kursaison begab sich Pat. nach Pjatigorsk, wo er 30 Schwefelbäder nahm und eine entsprechende Anzahl von Quecksilbereinreibungen ausführte. Ausserdem wurde Pat. die ganze Zeit über mit Elektrisation der Wirbelsäule und der unteren Extremitäten behandelt und nahm zum Schluss seines Aufenthalts im Kaukasus noch 26 Kohlensäurebäder in Kislowodsk.

Diese Behandlung hatte, nach den Aussagen des Patienten, einen bedeutenden Erfolg. Am Schluss seines Aufenthalts im Kaukasus konnte Pat. auf ebenen Wegen Spaziergänge machen, wenn er dieselben alle 5-10 Minuten unterbrach und sich erholte, im allgemeinen konnte er täglich bis zu 1/2 Werft gehen. Die Funktion der Harnblase besserte sich ebenfalls, wenn Pat, auch jetzt noch zeitweilig nur in sitzender Stellung Harn lassen konnte.

Eine solche Zunahme der Kräfte war hauptsächlich in den letzten 2 Wochen in Kislowodsk bemerbar und hat wohl auch nur dort bestanden. Schon 2 Wochen nach seiner Rückkehr nach Kiew konnte sich Pat, wieder nur mit. Mühe in seiner Wohnung bewegen.

2 Monate nach seiner Rückkehr nach Kislowodsk wurde bei Pat. während der Untersuchung in Bezug auf Muskelsinn und Reflexe (Hautund Sehnenreflexe) der Status quo ante konstatiert. Die Schmerz- und Temperaturempfindung war an beiden unteren Extremitäten unterhalb des Knies und im Gebiete des 4., 5. und 6. Interkostalnerven bedeutend herabgesetzt. Die Tastempfindung befand sich am Abdomen in einem Zustande von Hyperästhesie. Der Umfang der oberen und unteren Extremitäten hat nicht abgenommen. Die rohe Kraft der unteren Extremitäten ist so gering, dass der Pat. nicht imstande ist, die Beine im Knie zu beugen oder die Zehen zu heben, wenn der Finger des untersuchenden Arztes einen Gegendruck ausübt. Die galvanische und faradische Erregbarkeit der Muskeln weicht nicht von der Norm ab.

Der Gang ist ataktisch-paretisch. Pat. kann nur mit Mühe 10-15 Schritte machen und schleppt dabei die Fisse und wirft sie seitwärts.

In diesem Falle konnte die Diagnose "Tabes dorsalis" keinem Zweifel unterliegen.

4. Frau L. . . . swa, 45 Jahre alt, wandte sich am 10. Oktober 1900 an mich und klagte über Schwäche in den unteren Extremitäten. Bei der Untersuchung wurde Folgendes konstatiert: Gute Ernährung des ganzen Körpers. Auf der rechten Seite sind die Paresen der Muskelgruppen des Beckens und des Unterschenkels schärfer ausgeprägt als auf der linken Seite. Die elektrische Reaktion ist unverändert. Der Muskeltonus der unteren Extremitäten ist etwas matt. Der Muskeltonus der oberen Extremitäten weicht nicht von der Norm ab. Der Umfang des rechten Unterschenkels ist um $11 / 2 \mathrm{~cm}$ geringer als der des linken. Fibrilläres Zittern ist nicht vorhanden. Die Haut- und Sehnenreffexe sind vorhanden, aber matt. Die Empfindung an den unteren Extremitäten ist nur für Berührung herabgesetzt. Der Muskelsinn ist stark verändert. Eine Ataxie ist nur an den oberen Extremitäten leicht angedeutet. Das Rombergsche Symptom ist 
nicht vorhanden. Die Schmerz- und Tastempfindung ist nicht überall gleich, sondern nur im Verlauf der Nervenwurzeln stark herabgesetzt; eine gleichartige Verteilung der herabgesetzten Empfindung auf den Verlauf der Nervenwurzeln ist auch am Brustkorbe im Gebiete der Interkostalnerven bemerbar. Die Pupillen sird verengert. Beim Harnlassen fühlt Patientin das Bedürfnis mitzudrängen.

Patientin ist 15 Jahre verheirate1, hat keine Kinder gehabt. Der Mann der Patientin starb nach 12jähriger Ele an Paralysis progressiva. Patientin hat viele unruhige Zeiten und Gemütsbewegungen durchgemacht.

Die erwähnte Schwäche hat Patientin zum ersten Mal vor 1/2 Jahren bemerkt, als sie sich im Verlauf zweier Wintermonate während einer Periode starker Gemütsbewegungen in einem kalten und feuchten Raume aufhielt. Schmerzen und Parästhesien hat Patientin weder damals empfunden, noch empfindet sie sie jetzt. Einige Zeit, wahrscheinlich $1 \mathrm{Jahr}$, vor dem Auftreten der Schwäche in den unteren Extremitäten musste die Patientin beim Harnlassen stärker mitzupressen, und sie sah fixierte Gegenstände doppelt. Nach kurzer Zeit (3-4 Monaten) schwand das Doppeltsehen und die Beschwerden beim Harnlassen ohne jegliche ärztliche Massnahmen. Die Schwäche in den unteren Extremitäten nahm ebenfalls so weit ab, dass Patientin sich frei bewegen konnte.

Die gegenwärtige Erkrankung trat, in Form einer neien Schwächeattacke in den unteren Extremitäten nach Bädern in kaltem Flusswasser im Verlauf von 2 Wochen auf.

Die Diagnose von Tabes dorsalis im präataktischen Stadium machte im gegebenen Falle keine Schwierigkeiten, obgleich die typischen Schmerzen und Parästhesien fehlten.

Ebenso wie in den anderen Fällen zeichnete sich diese Periode der Erkrankung durch eine motorische Störung aus - durch eine schnell vorübergehende Parese der Muskelgruppen der unteren Extremitäten.

5. Am 15. September 1902 wurde die 30 jährige M. W. T. in die Frauenabteilung der Nervenklinik aufgenommen. Die Eltern der Patientin zeichneten sich durch einen schwächlichen Gesundheitszustand aus. Der Vater litt an einem Herzübel; die Mutter erkrankte mit 55 Jahren an einem Schlaganfall und hatte vorher 3 Aborte durchgemacht. Gegenwärtig zeigen sich bei der Mutter der Patientin Seltsamkeiten des Charakters und sogar gewisse psychische Störungen.

Patientin war in ihrer Kindheit schwächlich, blutarm und sehr nervös.

In der Kindheit hat sie Masern, Scharlach, Sumpffieber, Typhus und Keuchhusten durchgemacht. Mit ungefähr 6 Jahren fing Patientin an zu lernien. Anfälle und Krämpfe sind nicht vorgekommen. Der Charakter der Patientin ist ängstlich und unruhig.

Mit 21 Jahren heiratete Patientin und abortierte infolge eines Falles im ersten Jahre der Ehe. Der Mann der Patientin ist 5 Jahre älter als letztere und soll vollständig gesund sein, nur zeitweise leidet er an Zuckungen und ausstrahlenden Schmerzen im ganzen Körper, die wohl einen neurasthenischen Charakter haben. Vor 1 Jahre bemerkte Patientin eine schnelle Ermǘdung der unteren Extremitäten. Nur mit Mühe konnte sie bergauf 
gehen und nur mit grosser Anstrengung Treppen steigen. Die Füsse erschienen ungewöhnlich schwer und die Bew egungen der unteren Extremitäten und zwar ganz besonders der Füsse und Zehen gingen nur sehr langsam vor sich. Eine Schwellung der unteren Ertremitäten, Schmerzhaftigkeit der Gelenke und Muskeln waren weder vor noch nach dieser Epoche vorhanden. Herzklopfen oder Atemnot verspürte Patientin beim Gehen nicht. In der Dunkelheit konnte Patientin gut geben, was sie aber ebenso ermüdete wie das Gehen im Hellen. Einige Monate nachher hatte Patientin das Gefühl, als wenn sie Sand oder Watte unter den Füssen hätte. Nach einem halben Jahr hatte sie das Gefuhl, als wenn ihre Beine znsammengebunden wären, gleichzeitig stellten sich ausstrahlende Schmerzen und Korsettgefühl ein. Schon damals begann Patientin Beschwerden beim Harnlassen zu fühlen.

Am Ende dies ersten Jahres nach dem Beginn der Erkrankung, begann Patientin Unbequemlichkeiten beim Gehen und Stehen im Dunkeln zu verspüren. Die Sehkraft hat etwas abgenommen, aber Doppeltsehen bemerkte Patientin nicht.

Seit dem letzten Jahr kann Patientin gar nicht mehr gehen, da die Füsse unter ihrem Körpergewicht zusammenbrechen. In den letzten Monaten gesellte sich noch das Unvermögen, das Gleichgewicht zu halten, daza — sie kann nicht einmal mehr aufrecht sitzen. Abmagerung hat Patientin nicht bemerkt, es hat im Gegenteil im letzten Jahr das Körpergewicht zugenommon.

Die Behandlung, der sich die Patientin unterzogen hatte, bestand in Sonnen - und Wasserbädern. Bei dieser Behandlung fühlte sich Patientin nicht besser, sie bemerkte aber, dass ohne alle Therapie einige Male die rohe Kraft der unteren Extremitäten etwas zunahm

Gegenwärtig empfindet die Patientin eine allgemeine Schwäche und zwar ganz besonders in den unteren Extremitäten; sie kann nur mit Mühe 10-15 Schritte gehen, die Beine knicken hierbei ein, und Patientin muss sich setzen. Bei Gemütsbewegungen fühlt Patientin Ameisenlaufen, hat das Gefühl von Sand unter den Sohlen, von Zusammengeschnürtsein unterhalb der Knice und im Fussgelenk Korsettgefühl und der 4. und 5 . Finger beider Hände vertauben ihr, was sie jedoch nicht stört, Klavier zu spielen; nur zwingt sie bereits nach 5 Minuten eine starke Ermüdung dex Hand das Spielen aufzugeben. Im ganzen Körper fühlt Patientin nicht gerade starke ausstrahlende Schmerzen. Beim Harnlassen muss Patientin mitdrängen. Im Dunkeln kann sie nicht gehen.

Die Patientin ist von mittlerem Wuchs, gut gebaut und gut genährt; das Fettpolster ist gut entwickelt. Auf der Hant sind weder Ausschläge noch Narben bemerkbar. Die Haut ist leicht pigmentiert (Sonnenbäder). Die Lymphdrüsen des Nackens, des Halses, des Ellenbogens und der Leistengegend lassen sich nicht palpieren. Das Knochensystem ist normal. Die Gelenke der Extremitäten sind nicht geschwollen, nicht deformiert und eine Krepitation ist nicht bemerkbar. Ein Ödem des Unterhautzellgewebes ist nicht vorhanden. Die willkürlichen Bewegungen sind überall möglich; was aber die rohe Kraft anbetrifft, so ist sie an den unteren Extremitäten stark herabgesetzt. Die rechte Hand zeigt an dem Dynamometer 17, die linke 12. Patientin kann weder den Fuss heben noch das Knie beugen, wenn der Zeigefinger der untersuchenden Hand einen Gegendruck ausübt. 
An den Extremitäten ist die Empfindung für Berührungen leicht herabgesetzt. Die Schmerzempfindung ist im allgemeinen in Form von Längsstreifen an den oberen und unteren Extremitäten herabgesetzt. Das Gebiet der 1. und 2. hinteren Lendenwurzel ist normal, das Gebiet der 5. Wurzel aber ist hyperästhetisch und das Gebiet der 3. Lendenwurzel befindet sich im Zustande einer Hypästhesie. Im allgemeinen ist die Empfindung an der linken Extremität in geringerem MaBe gestört als an der rechten; am Rumpf und in den Interkostalräumen weicht die Schmerzempfindung nicht von der Norm ab. An der linken oberen Extremität ist eine diffuse Herabsetzung der Schmerzempfindung im Gebiet der 5., 6. und 7. hinteren Halswurzel bemerkbar; an der rechten oberen Extremität ist die Schmerzempfindung im Gebiet der ersten hinteren Brustwurzel herabgesetzt. Der Muskelsinn ist an den unteren Extremitäten stark verändert, an den oberen Extremitäten weicht derselbe nur wenig von der Norm ab. Die Wärmeempfindung ist im Gebiet der 3. und 4. hinteren Lendenwurzel an den Beinen stark herabgesetzt und zwar am rechten mehr als am linken. Die Gebiete der 1. und 2. Lendenwurzel befinden sich in Bezug auf Wärme in einem Zustande von Hyperästhesie. An den oberen Extremitäten ist die Wärmeempfindung an denselben Stellen herabgesetzt, an denen auch eine Störung der Schmerzempfindung besteht. Die Kälteempfinduug zeigt dieselben Abweichungen wie die Wärmeempfindung und zwar auch an den nämlichen Stellen.

Die Reflexe: Das Kitzeln der Fusssohle ruft keine reflektorischen Kontraktionen des Fusses hervor. Der mittlere Abdominalreflex ist matt, der obere und untere fehlen ganz; der Pharyngealreflex ist vorhanden; der Kornealreflex ist normal; der Reflex der Achillessehne, der Patella, des M. triceps und des M. supinator longus fehlen auf beiden Seiten; der Unterkieferreffex ist gut erhalten. Im Dunkeln sind beide Pupillen gleich weit; bei einfallendem Licht ist die linke Pupille weiter als die rechte, die sympathische Reaktion fehlt an der linken Pupille und ist an der rechten sehr matt; die Lichtreaktion fehlt am 'linken Auge und ist am rechten herabgesetzt; das Konvergieren der Gesichtsaxen ruft eine Verengerung der Pupillen hervor. Das Gesichtsfeld ist breiter als normal.

Die peripheren Nervenstämme sind bei Druck empfindlich; fibrilläres Zittern ist nicht bemerkbar. Muskelatrophien sind nicht vorhanden, die Konfiguration der einzelnen Muskeln ist normal. Die Herztöne sind nicht ganz rein; der Geruchssinn ist normal, die Geschmacksempfindungen ebenfalls. Die galvanische und faradische Erregbarkeit sowohl der Nerven als auch der Muskeln ist normal.

Im Dezember 1905 sah ich den Mann von Frau T. in der Heilanstalt von Dr. Korschun wegen der progressiven Paralyse.

Auch in diesem Falle befindet sich die Tabes dorsalis im präataktischen Stadium und es treten ebenfalls die motorischen Störungen in den Vordergrund. In der Anamnese und dem Status praesens dieses Falles finden wir, dass paretische Erscheinungen im Krankheitsbilde dominieren, mit ihnen beginnt die Erkrankung und auf dieselben beziehen sich die hauptsächlichsten Klagen der Patientin.

Die hier angeführten Beobachtungen sind in der Beziehung einander ähnlich, dass es alles Fälle von Tabes dorsalis in dem einen 
oder dem anderen Stadium dieser Erkrankung sind. Zu gunsten dieser Schlussfolgerung sprechen: 1. die typischen Empfindungsstörungen, 2. die Ataxie, 3. die Veränderungen des Pupillenreflexes, 4. die Beschwerden beim Harnlassen und 5. die Veränderungen in den Sehnenreflexen - die Herabsetzung oder Steigerung derselben.

Von grossem Interesse ist in all diesen Fällen, dass im ersten Stadium dieser Erkrankung Schmerzen, Parästhesieen und andere für das Anfangsstadium typische Symptome sehr schwach ansgeprägt sind oder sogar vollständig fehlen.

In den Vordergrund treten im Gegenteil Klagen über Schwäche und Abnahme der Kräfte, die im ganzen Körper, meistenteils aber nur in irgend einer, oder auch in zwei Extremitäten verspürt werden.

Die geschwächten Teile sind entweder etwas abgemagert, d. h. mässig und diffus atrophiert, oder die Konfiguration und der Umfang der Muskeln weicht nicht von der Norm ab. Der Muskeltonus ist geschwächt. Die elektrische Reaktion der Muskeln sowohl wie die der Nerven ist normal.

Die Verminderung der motorischen Funktionen ist über die ganze Extremität diffus verbreitet, oder beschränkt sich auf einzelne Muskelgruppen, ohne sich hierbei nach der anatomischen Verteilung der peripheren Nerven zu richten. Diese Schwäche erscheint auf den ersten Blick dem eigentlichen Bilde der Tabes dorsalis fremd. Wir sind daran gewöhnt, bei den Trägern dieser Krankheit in den ersten Stadien äusserst energische Kontraktionen einzelner Muskelgruppen zu beobachten, die ausserdem zuweilen sehr anbaltend sind, da die Tabetiker keine Muidigkeit empfinden. Hier haben wir aber Individuen vor uns, die beständig oder zeitweilig Schwäche empfinden und bei denen die Kontraktionen einzelner Muskelgruppen geschwächt sind.

Sehr interessant ist diese Affektion durch ibre Schwankungen. Die Verminderung der motorischen Funktionen bessert sich nach einem gewissen Zeitraum und wird unter dem Einfluss der eínen oder anderen Behandlungsmethode mehr oder weniger wieder hergestellt, um späterhin wieder mehr hervorzutreten.

Die Ursachen der hier beschriebenen Erscheinungen sind nicht leicht festzustellen. Mit vollem Recht können in allen den angeführten Fällen Erkrankungen der peripheren Nerven, des Knochensystems und speziell der Gelenke ausgeschlossen werden, die in allen Fällen freibeweglich und nicht schmerzhaft waren. Ebenso konnte die Ursache der motorischen Schwäche nicht in einer Erkrankung der Muskeln in Form einer Myositis oder Myopathie gesucht werden, da die Muskeln in der Mehrzahl der Fälle gut konfiguriert waren, keine Schwellung aufwiesen, nicht schmerzhaft waren und eine normale elektrische Re- 
aktion aufwiesen. Es konnte auch in unseren Fällen vollständig eine organische Erkrankung des Gehirns oder der Leitungsbahnen desselben und zwar der Pyramidenbahnen ausgeschlossen werden; in derartigen Fällen wären erhöhte Reflexe und spastische Erscheinungen vorhanden gewesen, in unseren Fällen aber sehen wir schwache Extremitäten und matte Reflexe oder aber sogar ein vollständiges Fehlen derselben. Die beschriebenen Störungen können auch nicht für irgend eine funktionelle Neurose, speziell für Hysterie gehalten werden, da objektive Daten hierfür in Form von Hemianästhesie, Fehlen des Pharyngealreflexes, Erhöhung der Reflexe, des entsprechenden Gemütszustandes etc. nicht beobachtet wurden.

In Übereinstimmung mit den oben zitierten Worten von $O$ p pen heim könnte man die in dem ataktischen und präataktischen Stadium der Tabes dorsalis entstehenden motorischen Störungen durch Ataxie erklären. Solch eine Annahme muss hier aber unbedingt verworfen werden.

Die ataktischen Bewegungen haben äusserst charakterische Merkmale: 1. Die einzelnen Exkursionen der ataktischen Extremitäten übersteigen die Norm, 2. die Richtung, in der sich diese Extremitäten bewegen, weicht rom Ziel ab, 3. die Bewegungen besitzen einen beschleunigten Rythmus und büssen 4. an Zweckmässigkeit ein, da ihre Arbeit eine intensivere ist, als es der Zweck erfordert.

Dieser Kategorie können die bezeichneten Erscheinungen bei den hier beschriebenen Patienten nicht zugezählt werden: 1. Die Exkursionen der unteren Extremitäten erreichen bei ihnen nicht einmal die Norm, 2. der Rythmus derselben ist verlangsamt, 3 . sie sind zweckentsprechend, obgleich sie das angestrebte Ziel nicht erreichen, und weichen 4 . insofern von der Richtung ab, als es ihnen an Energie gebricht, die Richtung einzuhalten.

Naturgemässer erscheint es, die hier erörterten Paresen und Paralysen einer Erkrankung der Zellen der Vorderhörner zuzuschreiben, die, wie es uns bekannt ist, schon mehrfach auch in der Gegenwart konstatiert worden ist (vgl. Literatur 5.7. 21) und dank der vervollkommneten Technik in den Frühstadien der Tabes dorsalis beobachtet wurde. Da die beschriebenen paretischen Erscheinungen aber die Neigung zeigten, sich zu bessern, so darf man die zu Grunde liegenden Veränderungen in den Zellen nicht als Degeneration derselben auffassen, sondern als eine Herabsetzung ihrer Funktionen, die einer Besserung fähig ist. Andererseits müssen hier ebenfalls wenig umfangreiche herdförmige Affektionen der Zellen angenommen werden, die durch eine kompensatorische Tätigkeit anderer benachbarter Teile der Vorderhörner ersetzt werden können.

Fin solcher Zustand der Zellen der Vorderhörner wurde auch 
experimentell an Tieren nach Durchschneidung der Hinterwurzeln beobachtet (vgl. 14. 21); die Veränderungen der Zellen traten hierbei in Form von einzelnen Herden auf und bestanden in einem Aufquellen des Zellkörpers, in einer gewissen Chromatolyse und in einer Lageveränderung des Kerns, ohne dass der letztere seine Form und Grösse veränderte. Die Metamorphose der Zelle war also sehr geringfügig, die Zelle konnte aber in diesem Zustande nicht als ganz normal betrachtet werden und ihre Funktionsfähigkeit musste im Verlauf einer gewissen Zeit fraglos abnehmen.

Wir halten es für durchaus möglich, bei unseren Patienten einen analogen Zustand der Zellenelemente der Vorderhörner anzunehmen, da sich dieselben unter den gleichen Verhältnissen befanden wie die soeben erwähnten Tiere, bei denen die Degeneration der Wurzelfasern durch das Experiment zustande gekommen war.

Wir beharren bei solch einer Annahme, da in der Literatur andere Tierexperimente bekannt sind, in denen sich nach Resektion der hinteren Wurzeln Paresen und Paralysen entwickelten und zuweilen eine gewisse Atrophie des affizierten Körperteiles bemerkbar war und dennoch die Funktionen sich ziemlich bald wiederherstellten.

Claude-Bernard durchschnitt beim Frosch und beim Hunde die hinteren Wurzeln des $\mathrm{N}$. ischiadicus und beobachtete hiernach eine Parese oder sogar eine Paralyse der dem Experiment unterzogenen Extremität.

Mott und Sherrington durchschnitten beim Affen die hinteren Wurzeln einer der oberen Extremitäten und bemerkten, dass das Tier den betreffenden Arm nicht mehr bei willkürlichen Bewegungen benutzte; der Arm war geschwächt und paretisch. Ganz besonders demonstrativ war diese Erscheinung, wenn die 3., 4., 5., 6., 7. und 8. Hals- und die 1., 2. und 3. Brustwurzel durchschnitten wurden. Bei solch einer Massenresektion büsste das Tier die Greifbewegungen vollständig ein. Bei einer geringen Anzahl von' resezierten Wurzeln waren diese paralytischen Erscheinungen mehr begrenzt. Wenn diese Störungen auch noch so stark waren, so nahmen sie dennoch nach Ablauf einer gewissen Zeit ab oder verschwanden sogar vollständig.

Hering durchschnitt die 3., 4., 5., 6., 7. und 8. Hals- und die 1. und 2. Brustwurzel und konnte sich bei der Beobachtung der Folgen dieser Operation davon überzeugen, dass die dem Experiment unterzogene Extremität vollständig ihre Kraft verlor.

Marg ulic $\mathrm{e}^{16}$ ) durchschnittbeim Affen alle hinteren W urzeln der Cauda equina bis zum 5 . Kreazbeinsegment inklusive und bemerkte hiernach, dass die rechte hintere Extremität bei schnellen Bewegungen zurückblieb.

Gleichartige Versuche wurden von Korniloff ausgeführt und auch 
er beobachtete paretische und paralytische Erscheinungen an einer der hinteren Extremitäten, deren hintere Wurzeln reseziert worden waren.

In allen diesen Fällen zeichneten sich die motorischen Störungen dadurch aus, dass sie nur kurze Zeit anhielten und nach einer gewissen, recht langen Zeitperiode abnahmen und vollständig verschwanden.

In einigen von diesen Versuchen wurde eine Herabsetzung der Ernährung der dem Experiment unterzogenen Extremität konstatiert, und zwar trat sie in Form von Muskelatrophien zutage.

Hering fand bei seinen Hunden nach Durchschneidung der hinteren Wurzeln eine Verminderung der Muskelmassen.

Dasselbe beobachteten Mott und Sherrington.

Die in diesen Fällen ausgeführte elektrische Reaktion ergab in den paretischen Extremitäten ein normales Resultat.

Diese Daten erlauben es uns, die Ursachen der beschriebenen Störungen besonders bei denjenigen von unseren Patienten leicht zu begreifen, bei denen ausser der Abnahme der motorischen Funktionen noch eine Muskelatrophie vorhanden war.

Wahrscheinlich liegt eine analoge Zellmetamorphose der Vorderhörner den Paresen bei unseren übrigen Patienten zugrunde, bei denen keine Muskelatrophie bemerkbar war. Vielleicht waren aber auch die Veränderungen der Zellen der Vorderhörner geringer, oder waren auf eine geringere Anzahl von Zellen ausgedehnt. Solch eine Schlussfolgerung würde aus der Gleichartigkeit der Erkrankung, an der alle hier angeführten Patienten litten, resultieren.

Ausser diesen, zu der Zahl der organischen gehörenden Ursachen müssen hier auch noch einige funktionelle Momente zugelassen werden, und zwar darf der Verlust derjenigen Reize, die von der Peripherie her durch die hinteren Wurzeln zu den Vorderhörnern gelangen und die Bedeutung von motorischen Impulsen haben, nicht ausser acht gelassen werden.

Raichline, Bruns, Pick und Bickel nahmen, ein jeder von seinem Gesichtspunkte ausgehend, an, dass die hierbei zustande kommende Störung der Empfindung die motorischen Funktionen durch Vermittelung der Grosshirnrinde beeinflusst. Das Tier, das eine unbestimmte Vorstellung von seiner anästhetischen Extremität hat, schliesst dieselbe vollständig aus seinen motorischen Assoziationen aus, benutzt sie zu seinen lokomotorischen Zwecken nicht und innerviert'dieselbe nicht genügend. Mit dieser Erklärung kann man sich teilweise einverstanden erklären, sie ist aber nicht für alle Fälle anwendbar.

Anton (17) beobachtete einen Patienten mit einseitiger Herabsetzung der Empfindung. Bei diesem Patienten bestanden äusserst charakteristische Bewegungen der linksseitigen anästhetischen Extre- 
mitäten. Ohne fremde Einwirkung hielt er die Extremitäten immer vollständig in Ruhe, wenn er aber gleichzeitig mit beiden Armen arbeitete, entwickelte er in ihnen eine mehr oder weniger gleiche Kraft.

Hering ( $\left.{ }^{15}\right)$ beobachtete einen Tabetiker mit äusserst stark herabgesetzter roher Kraft im rechten anästhetischen Arm; wenn aber dieser Patient beide Arme gleichzeitig anspannte, erwies sich der rechte stärker als der linke (S. 583).

Augenscheinlich kamen die geschwächten Bewegungen der anästhetischen Seite nicht infolge einer ungenügenden cerebralen Innervation und ungenügender Assoziationsreize zustande, denn sonst hätten die willkürlichen Bewegungen bei der Unmöglichkeit einer Kontrolle und eines Vergleiches (die linke Extremität ist tief anästhetisch) denselben Unterschied zu ungunsten der anästhetischen Extremitäten ergeben.

Hering ( $\left.{ }^{11}\right)$ gelangte zu dem Schluss, dass die Parese oder Paralyse einer bestimmten Bewegung unter solchen Bedingungen infolge einer zentripetalen Paralyse der sensiblen Nerven zustande kommt (Bd. 70. S. 582). Hering ist darin mit Sherrington einverstanden, dass jede hintere Wurzel demjenigen Muskel, der von der gleichnamigen vorderen Wurzel reagiert wird, zentripetale Nerven zusendet; infolge dessen paralysieren wir beim Durchschneiden der hinteren Wurzeln diejenigen Muskeln, die von diesen letzteren innerviert werden. Während des Experimentes kann man vorhersagen, welche Muskeln paralysiert sein werden, wenn man die Beziehung der vorderen Wurzeln zu ihnen kennt, und andererseits kann man die Paralyse der einen oder anderen Funktion vorhersagen, da es bekannt ist, welche Muskeln bei bestimmten koordinierten Bewegungen beteiligt sind.

Dieser Autor sieht die Ursachen der zentripetalen Lähmungen in einer Affektion der hinteren Wurzeln, wodurch die Stimula, die durch die hinteren Wurzeln zu den Vorderhörnern hinziehen, aufgehoben werden. Er findet, dass das Rückenmark, zo dem von der Peripherie her keine sensorischen Eindrücke mehr gelangen, in einen Ruhezustand versetzt wird, der dem Schlaf ähnlich ist. Hiervon überzeugte sich Hering in einer Reihe demonstrativer Versuche an Fröschen mit abgetragenem Grosshirn, die, wie bekannt, immer mit gekrümmtem Rücken und eingezogenen Extremitäten dasitzen. Die Versuche, die Lage solcher Tiere zu verändern, führen zu keinem Ziel, da sie sehr energische Bewegungen ausführen, um die früher gekrümmte Stellung wieder einzunehmev. Hering war in der Lage, den peripheren und sensorischen Ursprung dieser Bewegungen beweisen zu können, während er von ihm für rein spinal gehalten wnrde, d. h. 
selbständig in den Vorderhörnern des Rückenmarks entstanden. Beim Durchschneiden der hinteren Wurzeln solcher Frösche sah Hering ein Aufhören der Bewegungen der Extremitäten und des Rumpfes. Die Tiere, denen das Grosshirn entfernt war und bei denen gleichzeitig die hinteren Wurzeln reseziert waren, büssten die Fähigkeit ein, in ihre frühere Stellung zurückzukehren und lagen widerstandslos mit ausgestreckten Extremitäten da.

Es ist möglich, dass bei unseren Patienten die Wiederherstellung der rohen Kraft in den paretischen Extremitäten, die nach Behandlung mit der faradischen Bürste eintrat, gerade durch den starken Reiz bedingt wurde, der die schlummernden Zellen der Vorderhörner erweckte.

Die motorischen Störungen können also bei unseren Patienten durch zweifache Ursachen erklärt werden: 1. durch eine gewisse organische Affektion der Zellen des Vorderhorns, die vielleicht imstande war, nach einiger Zeit wieder zu verschwinden (oder es wurde die Funktion der affizierten Zellen von den Nachbarzellen übernommen); 2. durch eine Herabsetzung der Reize, die von der Peripherie her in das Rückenmark gelangen und den allgemeinen Tonus der Zellen der Vorderhörner erhöhen.

Da sowohl die eine als auch die andere Ursache durch eine primäre Affektion der hinteren Wurzelfäden bedingt wird, zwingt uns dieser Umstand dazu, das beschriebene Frühsymptom der Herabsetzung der motorischen Kraft zu den Erscheinungen einer Tabes dorsalis zu rechnen und diese Erscheinung nicht als besondere Erkrankungsform anzusehen.

Eine derartige Störung hätte nichts Merkwürdiges und Besonderes an sich, wenn sie bei bejahrteren Patienten mit sicher konstatierter Tabes dorsalis auftreten oder im letzten Stadium der Tabes dorsalis entstehen würde. Unter solchen Verbältnissen würde dieses Leiden keinerlei diagnostische Schwierigkeiten darbieten; da aber dieses Leiden im Gegenteil unbemerkt, wäbrend eines verhältnismässig befriedigenden Allgemeinzustandes des Patienten, ohne die geringsten Hindeutungen auf die Möglichkeit eines Vorhandenseins von Tabes dorsalis, entsteht, kann es den Anstoss zu den verschiedenartigsten Vermutungen geben, und es würde sich niemand dazu entschliessen, diese Affektion der motorischen Sphäre für den Beginn von Tabes dorsalis zu halten.

Das beschriebene Symptom ist bisher noch wenig erforscht. In der uns zugänglichen umfangreichen der Tabes dorsalis gewidmeten Literatur fanden wir nur einige diesbezügliche Hinweise:

Unter den französischen Autoren, die eine motorische Tabes erDeutsche Zeitschr. f. Nervenheilkunde. XXX. Bd. 
wähnen, führt Grasset (Leçons de clinique médicale; deuxième série. 1896. p. 319) einen Fall von Tabes dorsalis an, in dem sich die Symptome in zweifacher Richtung entwickelten: 1. empfand der Patient verschiedenartige Parästhesien und 2. war bei ihm die motorische Sphäre stark geschwächt: p. 319: „Au mois de juillet 1892 il commence par eprouver une sorte de constriction à la base de la poitrine; trois ou quatre jours après, il sent les jambes fléchir sous lui, la marche devient difficile et déjà incoordinée, actuellement ses membres inferieurs sont très faibles ou plutôt inhabiles"; p. 323: "Il s'agit là d'un cas de tabes exclusivement moteur à l' incoordination precoce". In dieser Mitteilung nennt Grasset den Fall von Tabes dorsalis - motorische Tabes. Aus einer solchen Benennung lässt sich schliessen, dass das Besondere dieses Falles hauptsächlich in der Affektion der motorischen Sphäre bestand.

An einer anderen Stelle seines interessanten Buches beschreibt Grasset die Entwicklung von Tabes bei einer Frau und teilt mit, dass bei dieser Patientin nach dem Verlassen des Hospitals alle Symptome, mit Ausnahme der Schwäche, verschwanden; ibidem p. 281: "Quand la malade sort de l'hôpital de Temis tous ces troubles ont disparu; mais les jambes restent faibles. Quoique l'élément essentiel du Tabes soit l'incoordination sans paralysie, on observe cepandant quelque-fois des vraies paralysies et très souvent une lassitude rapide ou de la faiblesse comme dans notre cas". $^{46}$

Sehr wichtig, nur sehr kurz gehalten ist die Mitteilung Frenkels (Neurol. Zentralbl. 1897. S. 924), dass er einen Fall von Tabes gesehen habe, in dem sich das Leiden äusserst schnell, im Verlauf von 24 Stunden, entwickelt hatte und in Form von paralytischen Erscheinungen zutage trat; erst später wurde es klar, dass diese Parese oder Paralyse der Anfang einer Tabes dorsalis war.

Ungeachtet dessen, dass dieses Symptom wenig bekannt ist, bietet es dennoch im allgemeinen Bilde der Tabes dorsalis nichts Unerwartetes. Jedenfalls ist es uns bekannt, dass Affektionen der motorischen Nerven des Auges z. B. sehr häufig im Beginn der Tabes dorsalis beobachtet werden und sogar zu den typischen Symptomen derselben gerechnet werden.

Das unbedeutende Material, über das wir im gegebenen Falle verfügen, erlaubt es uns nicht, den Versuch zu wagen, die nächstliegenden ätjologischen Ursachen, die diese frühzeitige Entwicklung der motorischen Störungen begünstigen, zu erklären.

Wir erlauben uns, auf Grund des oben Mitgeteilten folgenden Schluss zu ziehen: 
Es gibt Fälle von Tabes dorsalis, in denen die Anfangssymptome in Form von Paralysen oder Paresen der oberen oder unteren Extremitäten auftreten. Die Zugehörigkeit dieser Fälle zur Tabes dorsalis lässt sich zuweilen schon bei der ersten Untersuchung des Patienten konstatieren und zwar auf Grund der verschiedenen, einander parallel auftretenden Symptome, z. B. Störungen des Pupillenreflexes, Störungen beim Harnlassen, Ataxie usw. In einigen Fällen aber ist die genaue Diagnose äusserst schwierig zu stellen.

Die motorischen Störungen müssen in derartigen Fällen zur Zahl der tabetischen Symptome gerechnet werden, da man als ibre Ursache eine Affektion der hinteren Wurzeln annehmen muss.

\section{Literatur.}

1) Anton, Zur Lokalisation der Muskelsinnstörungen im Gehirn. Zeitschrift für Heillkunde. Bd. 14. 1893.

2) Bickel, Über den Einfluss der sensiblen Nerven und des Labyrinths anf die Bewegungen der Tiere. Pflügers Archiv. Bd. 67.

3) Dersel be, Die Bedentung der Sensibilität für den tierischen Organismus. a) Münchn. med. Wochenschr. 1898. Nr.6. b) Deutsche Zeitschrift für Nervenheilkunde 1898. Bd. 13.

4) Bruns, Neurol. Zentralbl. 1897. 924.

5) Charcot-Pierret, Altérations de la substance grise dans le tabes. Soc. biolog. 1871. IV. 22.

6) Claude-Bernard, Leçons sur la physiologie du système nerveux. 1863. Paris.

7) Condeleon, Atrophie musculaire pendant tabes. Thèse de Paris 1897.

8) Hering, Über die Bewegungslosigkeit des Rückenmarksfrosches nach Durchschneidung der hinteren Wurzeln. Pfügers Archiv. Bd. 54. 1893.

9) Dersel be, Beitr. zur experimentellen Analyse koordinierter Bewegungen. Pflügers Archiv. Bd. 70. 1898.

10) Derselbe, Über zentripetale Ataxie beim Menschen und beim Affen. Neur. Zentralbl. 1897. Nr. 23.

11) Dersebe, Über Bewegungsstörungen nach zentripetaler Lähmung. Arch. f. experiment. Pathol. u. Pharm. Bd. 38. 1896.

12) Frenkel, Neur. Zentralbl. 1897. S. 925.

13) Korniloff, Über die Veränderung der motorischen Funktion bei Störungen der Sensibilität. Zeitschr. f. Nervenheilk. 1897.

14) Lapinsky, Zur Frage des Verlaufes der Kollateralen der hinteren Wurzeln und der Veränderung der Zellen der Vorderhörner nach Durchschneidung der ersteren. Archiv f. Psychiatrie 1906. Newrologitscheskij Westnik 1902 (russisch).

15) Leyden, Über Beteiligung der motorischen Muskeln bei Tabes dorsalis. Zeitschr. f. prakt. Med. 1877.

16) Margulice, Monatsschr. f. Neurologie und Psychiatrie. Bd. 1. Heft 4. 
200 IX. Lapinsky, Einige wenig beschriebene Formen der Tabes dorsalis.

17) Mott und Sherrington, Experiments upon the influence of sensory nerves upon movement and nutrition of the limbs. Royal Society 1895 .

18) Pick, Uber die sogen. Conscience musculaire. Zeitschr. f. Psychologie und Physiologie der Sinnesorgane. 1893. Bd. 4.

19) Raichline, Neurol. Zentralbl. 1897. S. 924.

20) Sherrington, Experiments in Examination of the peripheral distribution of the fibres, of the posteriors Roots of some spinal nerves. Royal society proceed. Febr. 1897.

21) Schaffer, Über Nervenzellenveränderungen bei Tabes. Monatsschrift f. Psychiatrie 1898.

22) Wharrington, a) Of the structural alterations observed in nervecelle. Journ. of Physiol. Bd. 23. p. 112. b) Further observations on the structural alterations. Ibidem 24. Nr. 6 . 